\title{
Comparative Metabolomics Analysis and Radical Scavenging Activity of Saraca asoca (Roxb.) de Wilde Flowers in Different Stages of Maturity
}

\author{
Anindita Hazra, Susmita Das* \\ Phytochemistry and Pharmacognosy Laboratory, Department of Botany, University of Calcutta, 35, Ballygunge Circular Road, Kolkata - \\ 700019, West Bengal, India
}

Received: July 24, 2020; Revised: November 18, 2020; Accepted: December 10, 2020

\begin{abstract}
The flower extracts of Saraca asoca were evaluated in their three different phenological stages of flowering [bud (S1), mature (S2) and senescent (S3)] in terms of chemical composition and antioxidant activity. The GC/MS based fingerprinting led to identification of 85 metabolites, including 9 amino acids, 20 organic acids, 7 fatty acids, 20 sugar and sugar acids, 8 sugar alcohols, 13 phenols and phenolic acids and 8 others compounds. The three flowering stages showed prominent changes in their metabolite profile during the process of maturation of the flowering stages from bud to mature to senescence stages determined via GC/MS based metabolomics and chemometric approaches. The amounts and composition of metabolites in each stage showed statistically significant differences, which were reflected in their antioxidant capacities. The three phenological stages showed antioxidant activities in a dose dependent manner, but the senescent stage showed highest superoxide radical scavenging activity $\left(\mathrm{IC}_{50}=65.17 \pm 2.647 \mathrm{mg} / \mathrm{ml}\right)$ and metal chelating effect $(6.65 \pm 0.331 \mathrm{mg} / \mathrm{ml})$ in agreement with their high content of phenolic acids. These differences were strongly reproduced in the chemometric analyses (PCA, PLS-DA and s-PLS-DA), identifying the most distinctive features of the three flowering stages. This study might be beneficial to select the most potent flowering stage for incorporation in functional food.
\end{abstract}

Keywords: Saraca asoca, phenological stages, flowering, antioxidant, phytochemicals, functional food

\section{Introduction}

Since time immemorial, plant products or their derivatives and other natural resources are beneficial for the treatment of various illnesses. Mainly fruits and vegetables provide most of the phytonutrients in human diet. But flowers can also become an important source of bioactive components and can be added in human diet. Earlier flowers were mainly eaten for their therapeutic properties rather than their nutritional features. In the present time, several metabolomics studies revealed the presence of important bioactive molecules by metabolite profiling of wild and ornamental flowers. Wild flowers can also be an important source of low cost natural antioxidants, and many edible flowers are used as food additives to enhance color, flavor, taste and fragrance to food and drinks (Kelley et al., 2001; Pires et al., 2018)

Saraca asoca (Roxb.) de Wilde, known as Ashoka in West Bengal, India, belongs to the family Caesalpiniaceae and is a small evergreen tree. The plant is one of the most ancient plants known, mentioned in old Indian Ayurvedic manuscripts and is geographically distributed mainly in Asia and some parts of North America (Murthy et al., 2008). The medicinal properties of this plant are beneficial in several gynecological complications (Panchawat and Sisodia 2010). In India, married women are known to consume Ashoka flower buds as a ritual to protect their children and for several gynecological problems (Pradhan et al., 2009).

All parts of this plant especially barks, leaves, flowers and seeds are considered useful with high medicinal impact (Shukla et al., 2008). The flowers are therapeutically important part as these are used in the treatment of cancer, diabetes, hemorrhagic dysentery, several uterine disorders like menorrhagia, and also used in bleeding piles, bacillary dysentery, etc. During a Bengali Hindu ceremonial known as 'Ashoka - sasthi' the flower buds are taken orally by women. Only a few reports on phytochemical constituents of leaves and flowers of the plant have been published earlier (Pradhan et al., 2010) but no detailed qualitative and quantitative phytochemical analyses are found for flowers.

The flowers of Saraca asoca are the important part of many Ashoka rich herbal formulations. So far, it has been reported to contain tannins, flavonoids, saracasin, saracadin, waxy substances, carbohydrates, proteins and steroids (Saha et al., 2013). The presence of many fatty acids like oleic acid, palmitic acid, stearic acid, linoleic acid and linolenic acids, glucosides like quercetin-3-O-pD-glucoside, apigenin-7-O-p-D-glucoside, pelargonidin3,5-diglucoside and cyanidine-3,5-diglucoside, steroids such as p- and $\gamma$-sitosterols, flavonoids such as quercetin, leucocyanidin and polyphenols such as gallic acid and

\footnotetext{
* Corresponding author e-mail: susouravipar@gmail.com.
} 
ellagic acid have been reported also (Pradhan et al., 2009; Saha et al., 2013; Gupta et al., 2014).

Due to normal physiological processes and various xenobiotic factors by the process of membrane lipid and many other biomolecular peroxidations, Reactive Oxygen Species (ROS) like superoxide anion, hydroxyl radicals and hydrogen peroxide are generated. These ROS are associated in the etiology of several high risk ailments like cardiovascular disorders, coronary artery disease, stroke, rheumatoid arthritis, diabetes, hypertension and several types of carcinogenicity (Lefer and Granger 2000; Zahin et al., 2009). Free radicals prompt oxidative damages to biomolecules. Antioxidants and free radical scavengers from natural sources exert a significant role in protecting humans from several contagious infections, stress related pathologies and degenerative disorders (Feugang et al., 2006; Saha et al., 2018). The adverse effects of synthetic antioxidants like BHA (Butylated hydroxyl anisole) and BHT (Butylated hydroxyl toluene) used as synthetic additives in food stuff have already been experienced by modern humans that these synthetic antioxidants induce immense toxicity, carcinogenicity and causes DNA damage and other aberrations (Rajkumar et al., 2010). Recently, scientists are looking for diet derived antioxidants or naturally occurring antioxidants to replace all those synthetic antioxidants which are being restricted for their adverse impacts.

The plant metabolomics is an important tool to identify and quantify primary and secondary metabolites of natural products (Mishra et al., 2015; Pandey et al., 2015; Patel et al., 2016). Although plant primary metabolites are indispensable to perform life functions, the production of plant secondary metabolites are influenced by genotype, phenological stages and eco-physiological conditions (Marrelli et al., 2012). The phenological stage is considered as the most important determining factor of the quality and quantity of metabolites from dietary, nutritional and pharmaceutical point of view and thus holds immense importance (Marrelli et al., 2012).

Saraca asoca flowers are a potent reservoir of bioactive components, which may vary through the floral developmental stages. Studying the metabolite composition of this flower developmental stage associated with specific metabolism and free radical scavenging activity would be of enormous importance. However, to the best of our knowledge, so far no such research has been done on the metabolite profiling and radical scavenging analysis with respect to the different phenological / maturation stages of Saraca asoca flowers from bud, to mature to senescent stages to find out the most potent floral stage to be incorporated as functional food. Moreover, the present study may also deliver an understanding of the optimisation of the procurement of Saraca flowers and the maximization of bioactive potentials.

\section{Materials \& Methods}

\subsection{Plant material}

Saraca asoca flowers were procured from the garden of Burdwan University Campus, East Burdwan, West Bengal $\left(23^{\circ} 25^{\prime} \mathrm{N}, 8^{\circ} 84^{\prime} \mathrm{E}\right)$, India, during the season of spring time in the month of March, 2018, with an average day temperature ranges between $27^{\circ} \mathrm{C}$. to $30^{\circ} \mathrm{C}$. for the three different developmental / phenological stages [bud stage: flowers with closed petal (S1), mature stage: full flowering stage, stamens are extruded and the petals are fully exposed (S2) and senescent stage : stamens dried and shrivelled, just started to develop fruit and flower near to senescence, but still attached (S3) with the plant (Figure 1)]. All the three stages of flowers were collected in ample amounts from the same species of plant and at the same season and weather pattern.

After proper taxonomic identification of the plant (Voucher No. Phytopharma 332 1a), the flower samples of S1, S2 and S3 stages were shade - dried separately and the dried samples were then powdered with an electric grinder and sieved to make fine dried homogenous powder of three different stages.

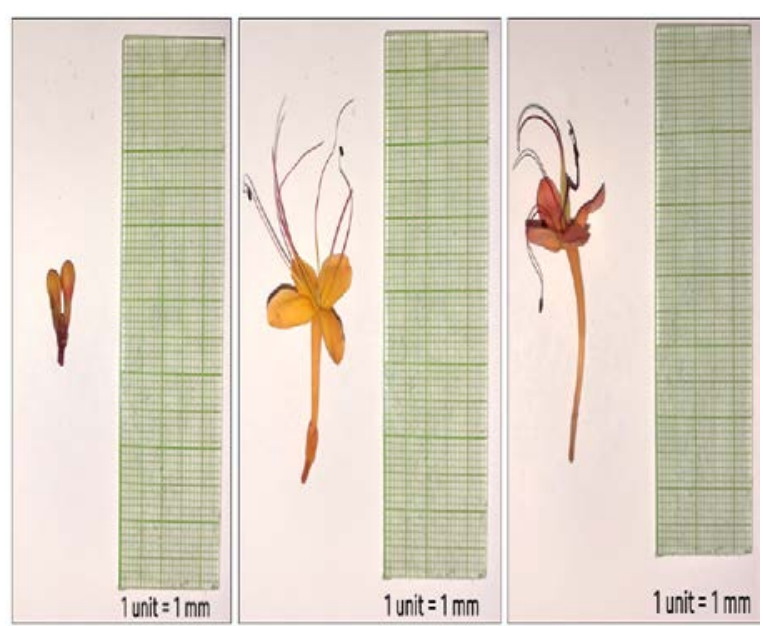

Figure 1. Flower of Saraca asoca in the S1, S2 and S3 stages

\subsection{Chemicals}

Adonitol, MOX, MSTFA, FAME standards were procured from Sigma Aldrich (St. Louis, MO); and pyridine from Merck Specialities Private Limited, India. DPPH was obtained from Sigma, USA. NBT, ferrozine were obtained from SRL PVT. Ltd., India. Methionine, riboflavin and EDTA were obtained from HiMedia Laboratories Limited. Ferric chloride, ammonium molybdate were obtained from Merck Specialities Pvt. Ltd. All the other reagents used for sample preparation were of analytical grade, and all the solvents used for GC/MS were of HPLC grade.

\subsection{Preparation of extracts for the evaluation of antioxidant properties}

$80 \%$ methanolic extracts were prepared from the dried samples of S1, S2 and S3 stages. The samples (150 gram) were extracted by stirring with $300 \mathrm{ml}$ of $80 \%$ methanol in a water bath at $65^{\circ} \mathrm{C}$ for 3 hours and subsequently after cooling, the filtrate was cold centrifuged at 10,000 rpm for 20 minutes. The combined $80 \%$ methanolic extracts were evaporated to dryness under reduced pressure. The crude extracts obtained were preserved at $-20^{\circ} \mathrm{C}$ freezer for further study.

The dried crude extracts were re-dissolved in methanol $(1 \mathrm{mg} / \mathrm{ml})$ for evaluation of antioxidant potentiality. The final solutions were further diluted to different concentrations to determine the bioactivity for S1, S2 and S3 stages of the samples in in vitro assays. Each 
experiment was performed thrice. The results were expressed in $\mathrm{IC}_{50}$ values (i.e., the concentration at which the samples showing $50 \%$ inhibition of free radicals).

\section{4. $D P P H$ radical scavenging activity}

DPPH free radical scavenging activities of S1, S2 and S3 extracts were assessed following the method described (Braca et al., 2001). $0.1 \mathrm{ml}$ of extract was added to $3 \mathrm{ml}$ of DPPH solution (0.004\% methanolic solution). After 30 minutes of incubation at room temperature, the absorbance was measured at $517 \mathrm{~nm}$ using a UV-VIS spectrophotometer. Lower absorbance values indicate greater free radical scavenging activity. The percentage inhibition activity of scavenging $\mathrm{DPPH}$ radical was calculated using the formula $\left[\left(\mathrm{A}_{0}-\mathrm{A}_{\mathrm{e}}\right) / \mathrm{A}_{0}\right] \times 100$; where $\mathrm{A}_{0}=$ absorbance of control reaction and $\mathrm{A}_{\mathrm{e}}=$ absorbance in presence of extract. All the tests were performed in triplicates and the results were averaged.

\subsection{Superoxide radical (O2-) scavenging activity}

Superoxide radical scavenging activity was measured following the method of Banerjee and De (Beauchamp and Fridovich 1971; Dasgupta and De 2004) in the riboflavinlight-NBT system. The superoxide anion radicals were made in $50 \mathrm{mM}$ sodium phosphate buffer ( $\mathrm{pH} 7.8$ ), $13 \mathrm{mM}$ methionine, $2 \mu \mathrm{M}$ riboflavin, $100 \mu \mathrm{M}$ EDTA and $75 \mu \mathrm{M}$ NBT solution and $1 \mathrm{ml}$ extract of sample of different concentrations is added to the mixture. After 10 minutes of illumination from fluorescent lamp, the formation of blue formazan was measured by following the increase in absorbance at $560 \mathrm{~nm}$. One set of reaction tubes was covered with aluminium foil. Similar tubes with reaction mixture were kept in the dark and served as blanks. The percentage inhibition activity of scavenging superoxide radical was calculated with the formula $\left[\left(\mathrm{A}_{0}-\mathrm{A}_{\mathrm{e}}\right) / \mathrm{A}_{\mathrm{e}}\right]$ $\times 100$.

\subsection{Metal chelating effect (Ferrous ion)}

$\mathrm{Fe}^{2+}$ chelating ability proves the antioxidant capacity of plant extract (Wang et al., 2003). The reaction mixture containing $100 \mu \mathrm{l}$ of the plant extract, $200 \mu \mathrm{l}$ of $0.5 \mathrm{mM}$ ferrous chloride, $200 \mu \mathrm{l}$ of $5 \mathrm{mM}$ ferrozine were incubated at $37^{\circ} \mathrm{C}$ for 10 minutes. After addition of $1.5 \mathrm{ml}$ double distilled water to the mixture, the absorbance of the solution was taken at $562 \mathrm{~nm}$ where the lower absorbance indicated the stronger chelating effect.

\subsection{Total antioxidant capacity (TAC)}

The reaction mixture contained $0.1 \mathrm{ml}$ of sample solution and $1 \mathrm{ml}$ of reagent mixtures (prepared by mixing phosphate buffer, sulphuric acid and ammonium heptamolybdate in a ratio of 4:3:3) (Aguilar et al., 1999). The reaction mixture was incubated at $95^{\circ} \mathrm{C}$ for $90 \mathrm{~min}$., then the mixture was cooled to room temperature. The absorbance of the solution was measured at $695 \mathrm{~nm}$ against the blank. The reduction of $\mathrm{Mo}_{\mathrm{VI}}$ to $\mathrm{Mo}_{\mathrm{v}}$ by the extract and the formation of green phosphate / $\mathrm{Mo}_{\mathrm{v}}$ complex at acidic $\mathrm{pH}$ were assayed. The TAC was measured from the regression equation $\mathrm{y}=31.54 \mathrm{x}-0.001$ as equivalent to ascorbic acid.

\section{Sample preparation for GC/MS analysis}

$5 \mathrm{mg}$ of crude extract was dissolved in methanol: water in a ratio of 1:1. In it $20 \mu \mathrm{l}$ of ribitol (Adonitol - Internal
Standard) $(0.2 \mathrm{mg} / \mathrm{ml})$ was added and the aliquot made thereafter was distributed into eppendorff tubes $(50 \mu \mathrm{l} \times 4)$ and evaporated to dryness. The residue obtained was resuspended in $5 \mu \mathrm{l}$ of MOX ( $20 \mathrm{mg} / \mathrm{ml}$ in pyridine) and then shaken for 90 minutes at $30^{\circ} \mathrm{C}$. Then $45 \mu$ l of MSTFA was added and shaken at $37^{\circ} \mathrm{C}$ for 30 minutes for trimethylsilylation of acidic protons to enhance the volatility of components. $1 \mu \mathrm{l}$ of FAME markers (a mixture of IRI markers) was added (prepared using fatty acid methyl esters of C8, C10, C12, C16, C18, C20, C22, C24 and C26 linear chain length dissolved in chloroform). GC/MS analysis (Agilent 7890 A GC equipped with 5795 C inert MSD with Triple Axis Detector) was carried out following the method of Kind et al., 2009 (Kind et al., 2009) with some modifications (Das et al., 2016). Prior to injection in GC the samples were preserved at $4^{\circ} \mathrm{C}$ for 10 minutes to maintain sedimentation of components.

\subsection{Detection of metabolites by GC/MS}

The detection of metabolites was done using DB-5-MS capillary column under the following oven temperature programme: oven ramp $60^{\circ} \mathrm{C}$ ( 1 minute hold) to $325^{\circ} \mathrm{C}$ at10 ${ }^{\circ} \mathrm{C} / \mathrm{min}$, 10-minute hold before cool down, 37.5 minute run time. The injection temperature was set at $250^{\circ}$ $\mathrm{C}$, the MS transferline at $290^{\circ} \mathrm{C}$, and the ion source at 230 ${ }^{\circ} \mathrm{C}$. Helium was used as the carrier gas at a constant flow rate of $2.5 \mathrm{ml} /$ minute (carrier linear velocity 57.95 $\mathrm{cm} / \mathrm{sec}$ ). $1 \mu \mathrm{l}$ of sample was injected manually via the split mode (split ratio 1:5) onto the GC column. Before analysis, the method was calibrated with the FAME standards available on the Fiehn GC/MS Metabolomics library (2008) (Agilent Chem Station, Agilent Technologies Inc., Wilmington, USA) following user's guide. AMDIS was used to deconvolute GC/MS results to identify the chromatographic peaks. Auto-tuning and tune evaluation of Mass Detector was done at least once a week.

Identification of metabolites was done by comparing the RT, RI of the metabolites and also by comparing the MS fragmentation patterns of the mass spectra with the entries of compound in Agilent Fiehn GC/MS Metabolomics library (2008) using metabolite database AMDIS using Agilent RTL method. RT of some of the metabolites were further compared with that of the standard compounds.

\subsection{Statistical analysis}

Antioxidant activities were estimated in triplicates. The results were expressed as means \pm standard deviations. All the statistical tests were performed at 5\% significance level using Microsoft Excel 2013.

The three selected phenological stages were subjected to Multivariate Analysis to determine the differences in metabolite composition among S1, S2 and S3 stages.

To identify the statistical significance difference and to compare the dependent variables, the one-way ANOVA and a post-hoc test using Tukey's HSD were done and determined by p-values lower than 0.05 . Based on the generalized logarithm transformed dataset, heat map, clustering of samples as well as metabolites were also developed utilizing squared Euclidean distance and ward linkage.

The relative response ratios of the metabolites of S1, S2 and S3 stages were subjected to Metaboanalyst 4.0. PCA, 
PLS-DA and s-PLS-DA were applied as pattern recognition unsupervised classification method. The construction of the matrix for PCA included 85 variables (corresponding to the responses of metabolites) and 3 samples (corresponding to the flowering stages).

\section{Results}

\subsection{Evaluation of antioxidant potentialities}

In this investigation, the antioxidant activities of the studied flowering stages were measured and compared by DPPH, superoxide radical scavenging, metal chelation activity and TACs. All the flowering stages showed antioxidant activities. DPPH (1, 1-diphenyl-2picrylhydrazyl) is a widely known stable radical used for the assessment of the ability of phenolic substances which can donate labile $\mathrm{H}$-atom to the free radicals. In this assay, the H-donating antioxidant component reduces the DPPH to form non-radical DPPH-H (Shen et al., 2010). DPPH free radical scavenging activities of extracts of S1, S2 and S3 stages of Saraca asoca flowers were found proportionate to the concentration of the extracts $(r>0.9)$. Activity of S1 stage was found significantly higher than S2 and S3 stages in DPPH inhibition. IC $_{50}$ values are shown in Table 1. S1 stage (bud stage) showed the highest radical scavenging activity against $\mathrm{DPPH}\left(\mathrm{IC}_{50}\right.$ value $=65.82$ $\mathrm{mg} / \mathrm{ml} \pm 0.614$ ).

In this study, we have observed that the extracts of all the three stages of flowering scavenged the superoxide radical in a dose dependent manner $(r>0.93)$. $\mathrm{IC}_{50}$ values are presented in Table 1 . The S3 stage was significantly different from S1 and S2 stages. The S3 stage (senescent stage) gave highest inhibitory activity against superoxide radical $\left(\mathrm{IC}_{50}\right.$ value $\left.=65.17 \mathrm{mg} / \mathrm{ml} \pm 2.647\right)$.

The metal chelation effect is based on the chelation of ferrous ions by plant extract. Ferrozine can form complexes with ferrous ions $\left(\mathrm{Fe}^{2+}\right)$. The formation of complex with $\mathrm{Fe}^{2+}$ is disrupted in presence of plant extract as chelating agent resulting in a significant decrease in the red color of the complex. Removal or reduction of iron ion from the cellular system is a promising approach to prevent oxidative stress related diseases and disorders. $\mathrm{Fe}^{2+}$ ion chelating property of S1, S2 and S3 stages were compared as shown in Table 1. The ferrous ion chelating property was also found greatest in S3 stage $(6.65 \mathrm{mg} / \mathrm{ml} \pm$ 0.331 ).

No significant difference was estimated in TACs of the three stages of flowering (Table 1).

Table 1. Antioxidant activity of $80 \%$ methanolic extracts obtained from three stages of flowering (S1, S2 and S3) of Saraca asoca (Mean \pm $\mathrm{SE})$

\begin{tabular}{|c|c|c|c|c|c|}
\hline & & & Antioxidant assays $\left(\mathrm{IC}_{50}\right.$ values* & $\pm \mathrm{SE}) \mathrm{mg} / \mathrm{ml}$ & \\
\hline & & DPPH radical & Superoxide radical & Metal & Total Antioxidant \\
\hline & & scavenging activity & scavenging activity & Chelating Effect & Capacity \\
\hline \multirow[t]{3}{*}{ Flowering stage } & S1 (bud stage) & $65.82 \pm 0.614$ & $74.79 \pm 5.258$ & $15.161 \pm 0.43$ & $0.000305 \pm 3.295 \mathrm{E}-05$ \\
\hline & S2 (mature stage) & $95.26 \pm 6.225$ & $120.61 \pm 5.279$ & $12.905 \pm 0.129$ & $0.00037 \pm 7.33$ E-05 \\
\hline & S3 (senescent stage) & $71.39 \pm 1.711$ & $65.17 \pm 2.647$ & $6.65 \pm 0.331$ & $0.00041 \pm 8.758$ E-05 \\
\hline p-value** & & 0.0021 & 0.0004 & 5.05E-06 & \\
\hline
\end{tabular}

* $\mathrm{IC}_{50}$ values correspond to the sample concentration showing $50 \%$ of inhibitory activity

${ }^{* *}$ p value $<0.05$ indicates that the mean value of the evaluated parameters of one flowering stage differs from the others (ANOVA post hoc HSD Tukey's Test were performed)

\subsection{Characterization of metabolites}

A total of 85 metabolites have been identified (Table 2) following GC/MS based metabolomics approach from the three phenological stages of Saraca asoca flowers. The metabolites identified include 9 amino acids, 20 organic acids, 7 fatty acids, 20 sugar and sugar acids, 8 sugar alcohols, 13 phenols and phenolic acids and 8 other compounds using Agilent Fiehn Metabolomics Library.

The Relative response ratios (RRR) were calculated by normalization of the peak areas of each metabolite obtained by dividing with the weight of the sample and the peak areas of the internal standard. The relative response ratios correspond with the semi-quantitative concentration of each metabolite. From the log of RRR / gram sample extracted, dendrograms (Figure 2) and heat map (Figure 3) were prepared using Metaboanalyst 4.0 software to separate the three phenological stages of flowering based on the metabolite profiling. The data were further analyzed by unsupervised PCA (figure not given) and supervised discriminant analysis (PLS-DA and s-PLS-DA). PCA, PLS-DA and s-PLS-DA (Figure 4) separated the bud, mature and senescent stages distinctly from each other. 
Table 2.Tentative identification and quantification of metabolites

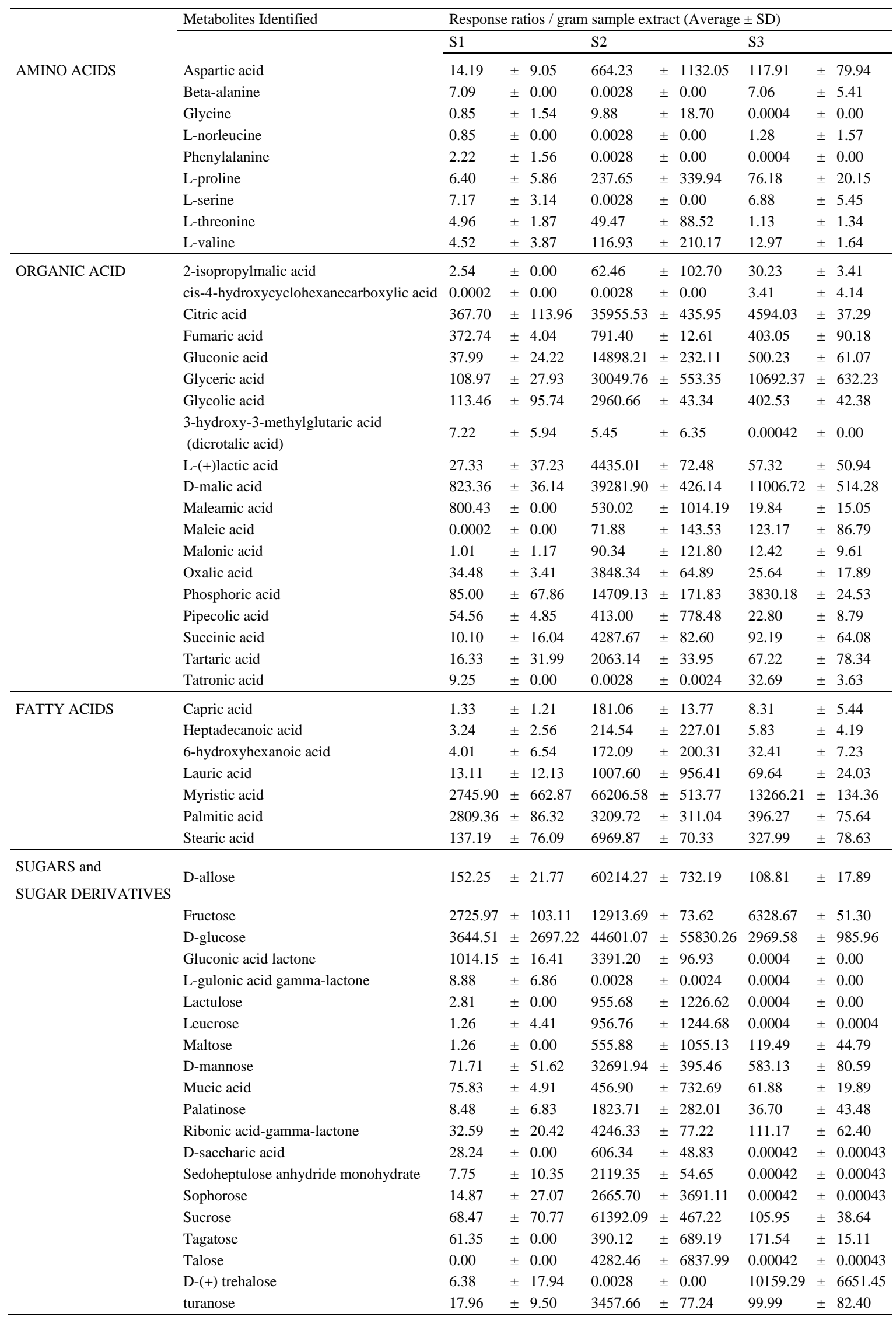




\begin{tabular}{|c|c|c|c|c|c|c|c|c|}
\hline \multirow[t]{8}{*}{ SUGAR ALCOHOLS } & Acetol & 11.58 & \pm & 0.00 & 0.0028 & \pm 0.00 & 2499.76 & \pm 874.17 \\
\hline & Allo-inositol & 169.24 & \pm & 70.83 & 29142.81 & \pm 344.88 & 3177.24 & \pm 246.06 \\
\hline & Galactinol & 170.14 & \pm & 2.39 & 53.24 & \pm 102.46 & 0.0004 & \pm 0.0004 \\
\hline & Glycerol & 0.89 & \pm & 0.00 & 16455.97 & \pm 19856.48 & 556.60 & \pm 234.03 \\
\hline & D-mannitol & 2.14 & \pm & 6.31 & 2365.66 & \pm 991.42 & 1644.39 & \pm 181.18 \\
\hline & Myo-inositol & 565.92 & \pm & 17.75 & 49024.79 & \pm 623.02 & 16995.64 & \pm 153.38 \\
\hline & D-sorbitol & 563.78 & \pm & 0.00 & 0.0028 & \pm 0.00 & 69.40 & \pm 55.82 \\
\hline & D-threitol & 0.00017 & \pm & 0.00 & 166.05 & \pm 304.29 & 461.06 & \pm 74.13 \\
\hline \multirow{14}{*}{$\begin{array}{l}\text { PHENOLS AND } \\
\text { PHENOL DERIVATIVES }\end{array}$} & O-acetylsalisylic acid & 0.00017 & \pm & 0.00 & 95.43 & \pm 175.36 & 24.72 & \pm 19.63 \\
\hline & & & & & & & & \\
\hline & Alizarin & 1.60 & \pm & 3.55 & 38.14 & \pm 49.89 & 0.0004 & \pm 0.0004 \\
\hline & Benzoic acid & 1.79 & \pm & 0.55 & 0.0028 & \pm 0.00 & 5.85 & \pm 1.67 \\
\hline & 3,4-dihydroxybenzoic acid & 9.44 & \pm & 9.27 & 0.00283 & \pm 0.00 & 0.00042 & \pm 0.00043 \\
\hline & Gallic acid & 1297.04 & \pm & 33.74 & 43236.67 & \pm 440.85 & 11015.00 & \pm 68.58 \\
\hline & Gentisic acid & 1287.79 & \pm & 0.00 & 135.04 & \pm 27.75 & 60.16 & \pm 85.69 \\
\hline & Homogentisic acid & 0.0002 & \pm & 0.00 & 0.0028 & \pm 0.00 & 1.56 & \pm 1.13 \\
\hline & 4-hydroxy-3-methylbenzoic acid & 0.00017 & \pm & 0.00 & 0.00283 & \pm 0.00 & 19.46 & \pm 22.57 \\
\hline & 2-hydroxyphenylacetic acid & 0.00017 & \pm & 0.00 & 0.00283 & \pm 0.00 & 6.35 & \pm 7.39 \\
\hline & Hydroquinone & 0.17 & \pm & 0.58 & 23.24 & \pm 32.75 & 0.00042 & \pm 0.00043 \\
\hline & 4-isopropylbenzoic acid & 0.96 & \pm & 1.11 & 110.88 & \pm 163.74 & 5.99 & \pm 0.97 \\
\hline & Shikimic acid & 46.94 & \pm & 50.95 & 1586.94 & \pm 2409.22 & 907.11 & \pm 160.31 \\
\hline & 4-vinylphenol & 46.55 & \pm & 0.42 & 20.34 & \pm 32.87 & 0.00042 & \pm 0.00043 \\
\hline \multirow[t]{8}{*}{ N-BASES AND OTHERS } & Adenosine & 4.39 & \pm & 5.74 & 344.05 & \pm 55.30 & 0.00042 & \pm 0.00043 \\
\hline & Adrenaline & 4.00 & \pm & 0.00 & 197.65 & \pm 34.00 & 0.00042 & \pm 0.00043 \\
\hline & Nicotinic acid & 1.77 & \pm & 6.31 & 232.04 & \pm 38.78 & 16.62 & \pm 4.46 \\
\hline & Pyrogallol & 5.48 & \pm & 3.11 & 166.47 & \pm 65.77 & 0.00042 & \pm 0.00043 \\
\hline & Thymine & 3.71 & \pm & 0.00 & 16.61 & \pm 3.55 & 0.97 & \pm 1.12 \\
\hline & Tyramine & 66.34 & \pm & 50.16 & 731.81 & \pm 83.42 & 106.56 & \pm 6.33 \\
\hline & Uracil & 67.74 & \pm & 2.31 & 153.56 & \pm 184.28 & 14.49 & \pm 12.20 \\
\hline & Urea & 2.04 & \pm & 1.33 & 0.0028 & \pm 0.0024 & 0.0004 & \pm 0.0004 \\
\hline
\end{tabular}

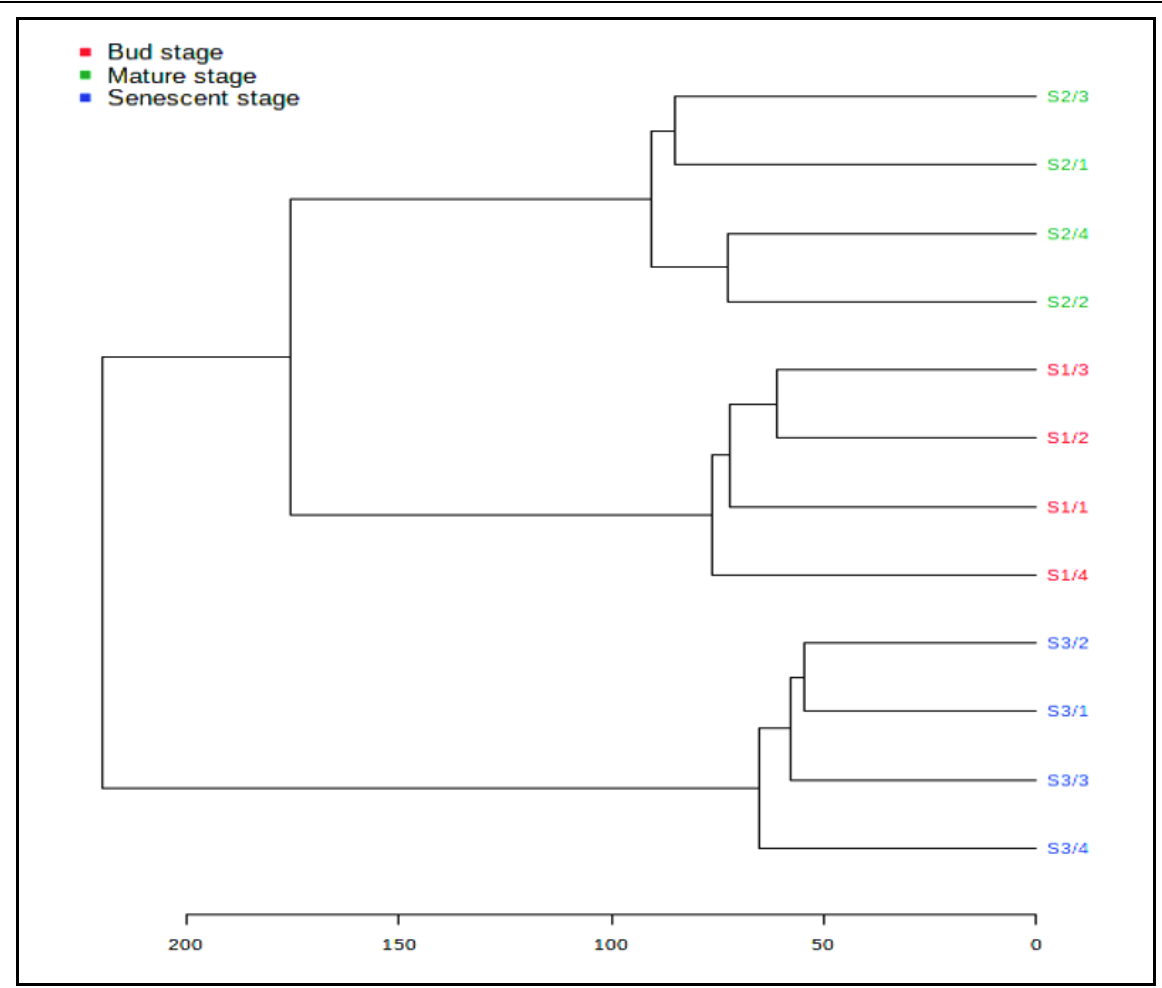

Figure 2. Dendrogram generated from log of normalised RRRs of three phenological stages of flowering of Saraca asoca 


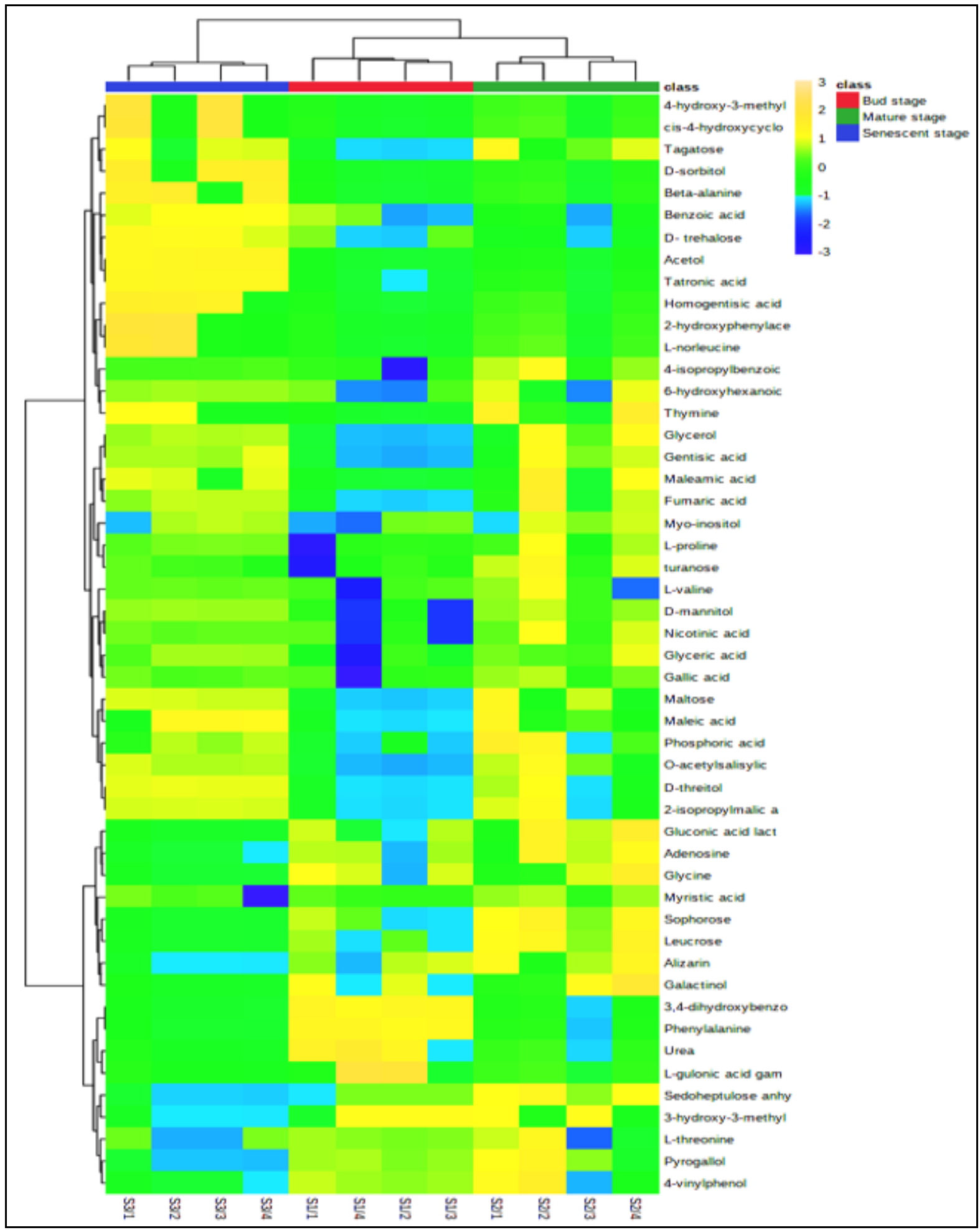

Figure 3. Heat map showing changes in relative concentration among the three phenological stages of flowering

The dendrogram (Figure 2) and the heat map (Figure 3) illustrate that the three flowering stages (Bud-S1, MatureS2 and Senescent-S3) of Saraca asoca are distinctly different based on their primary and secondary metabolite compositions. In the heat map (Figure 3), the changes in relative concentration of the identified metabolites among the three stages of flowering have also been well characterized distinctly.

\section{Discussion}

Metabolomic approaches, utilizing GC/MS and other mass spectrometric analyses allow the single experiment profiling and semi-quantification and or quantification of numerous metabolites that are generally conserved across the kingdoms of life (Tohge and Fernie 2014). Metabolomics can take a print of any biochemical status to evaluate biochemical changes in metabolic pools (Hanhineva et al., 2008; Osorio et al., 2014). The large 
data sets obtained in metabolomic experiments are analyzed with multivariate statistical tools in an aim to determine biological components that show differential behavior under various circumstances within a single species.

In this study among the 85 identified metabolites, important metabolites viz., the top 25 metabolites based on VIP (Variable importance projection) scores detected by PLS-DA loading plots (Figure 5) for the separation of S1,

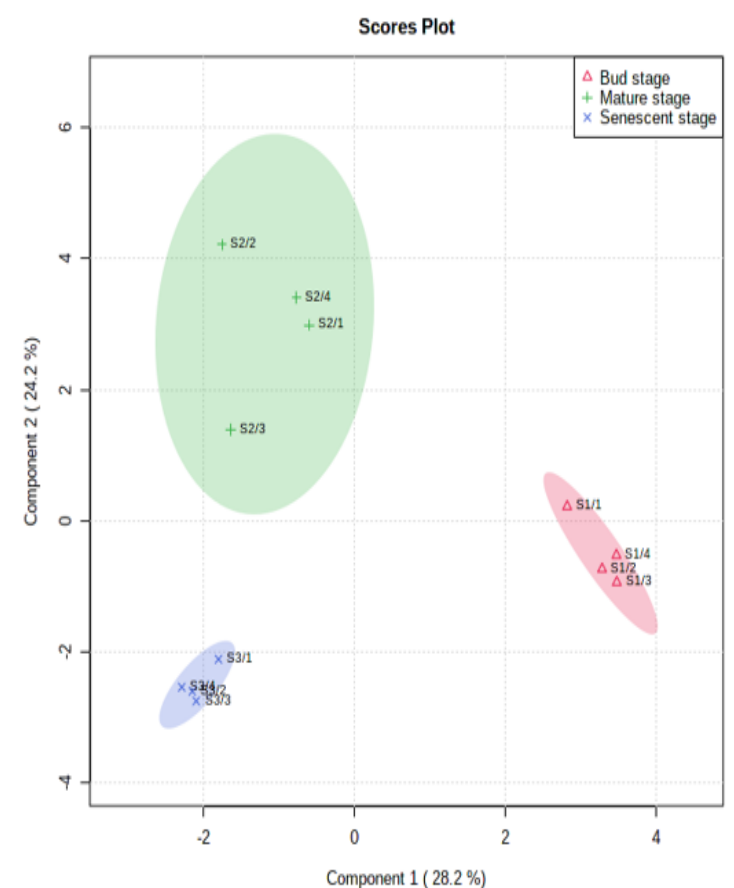

S2 and S3 stages were found: acetol, glycerol, D-threitol, maltose, tartronic acid, gentisic acid, 2-isopropyl malic acid, O-acetylsalisylic acid, D-trehalose, maleic acid, Dmannitol, tagatose, 3,4-dihydroxy benzoic acid, D-sorbitol, pyrogallol, maleamic acid, phenylalanine, glyceric acid, beta-alanine, sedoheptulose anhydride monohydrate, 3hydroxy-3-methylglutaric acid (dicrotalic acid), 4vinylphenol, homogentisic acid, 6-hydroxyhexanoic acid and adenosine.

Figure 4. s-PLS-DA 2-D and 3-D scores plot indicating each flowering stage is individualized based on their metabolite profile

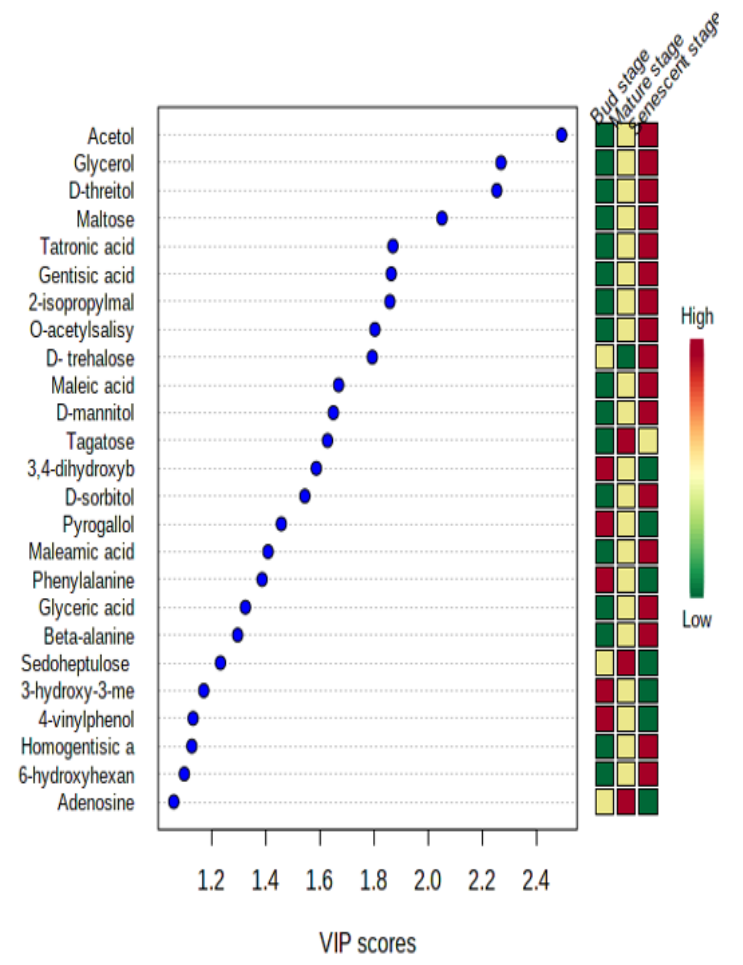

Figure 5. Loading plot in PLS-DA showing the top 25 metabolites
The bi-plot (Figure 6) of component loadings also indicates the distinct groups corresponding to each flowering stage (S1, S2 and S3) based on their different chemical profile. The flowering stage corresponding to S1 is mainly characterized by high contents of phenol derivatives like 3-hydroxy-3-methylglutaric acid (Dicrotalic acid), 3, 4-dihydroxybenzoic acid; amino acids like L-serine, phenylalanine; sugar derivative like Lgulonic acid gamma lactone and urea. On the other hand, the group corresponding to S2 is distinguished clearly by having high amounts of sugars like sophorose, leucrose, lactulose, talose, D-allose and sugar derivatives like gluconic acid lactone, sedoheptulose anhydride monohydrate, D-saccharic acid, sugar alcohols like galactinol; phenolic compounds like 4-vinylphenol, hydroquinone, pyrogallol, alizarin; organic acids like oxalic acid, tartaric acid, maleic acid and a few other compounds like adenosine, adrenaline, whereas the group corresponding to S3 is characterised and distinguished by having a large number of phenolics such as benzoic acid, gentisic acid, gallic acid, O-acetylsalicylic acid, 4hydroxy-3-methyl benzoic acid, homogentisic acid; sugars like D-trehalose, maltose, tagatose; sugar alcohols such as acetol, D-sorbitol, glycerol, D-threitol, D-mannitol, myoinositol; organic acids like maleamic acid, tartronic acid, glyceric acid, isopropylmalic acid, acetic acid, maleic acid; amino acids such as beta-alanine, L-valine, norleucine and other compounds like nicotinic acid, thymine, etc. 


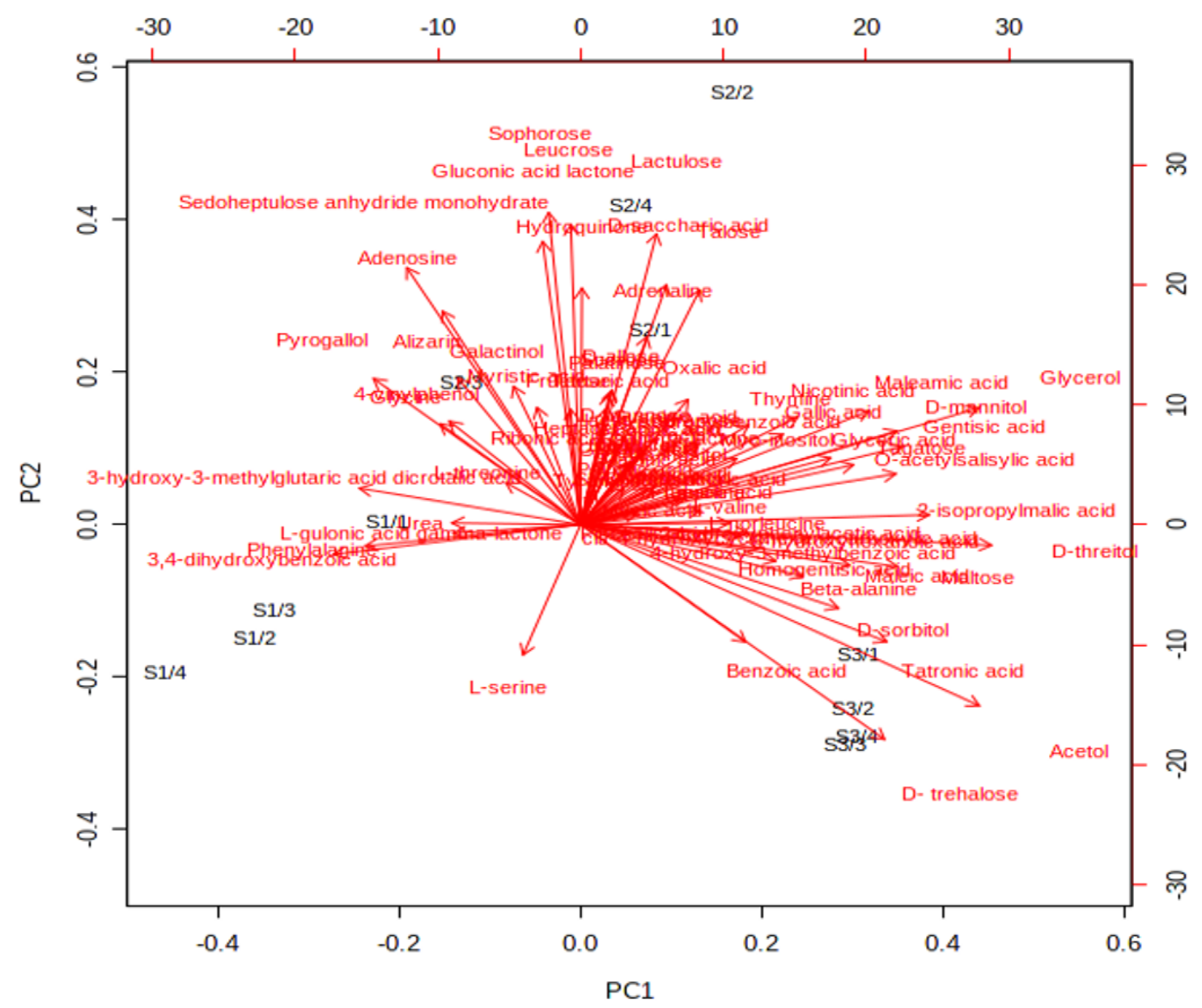

Figure 6. Biplot of component loadings using flowering stage as labelling variable

\section{Conclusion}

The three flowering stages showed prominent changes in their metabolite profile during the process of maturation of the flowering stages from bud to mature to senescence stages determined via GC/MS based metabolomics and chemometric approaches. The amounts and composition of metabolites in each stage showed statistically significant differences, which were reflected in their antioxidant capacities. Although all the three phenological stages showed antioxidant activities in a dose dependent manner, the senescent stage showed highest superoxide radical scavenging activity $\left(\mathrm{IC}_{50}=65.17 \pm 2.647 \mathrm{mg} / \mathrm{ml}\right)$ and metal chelating effect $\left(\mathrm{IC}_{50}=6.65 \pm 0.331 \mathrm{mg} / \mathrm{ml}\right)$ in agreement with their high content of phenolic acids. These differences were strongly reproduced in the chemometric analyses (PCA, PLS-DA and s-PLS-DA). It could also be reflected in the distribution of the biplot markers in different groups or clusters (which correspond to each phenological stage of flowering of Saraca asoca) with significant differences in an integrated manner. The differences detected might be beneficial for the selection of a specific flowering stage concerning its incorporation as an important source of medicine and also as a functional food.

\section{Acknowledgements}

The authors are grateful to Burdwan University, West Bengal, for granting permission to collect the samples in ample amounts. GC-MS based work was supported by DST-FIST Program, Govt. of India [Grant Number: SR/FST/LS1-459/2010], conducted in the Central Instrumentation Facility, Department of Botany, University of Calcutta, West Bengal, India.

\section{Authors' contributions}

SD and AH conceived the research project and assisted in editing; $\mathrm{AH}$ conducted the research, $\mathrm{SD}$ analyzed the results and wrote the manuscript.

\section{Data availability}

Most of the data not explicitly presented are available in the Supplementary Material. The remaining is available upon request (susouravipar@gmail.com).

\section{Conflict of interest}

The authors declare that they have no conflict of interest. 


\section{References}

Aguilar Urbano M, Pineda Priego M, Prieto P. 1999 Spectrophotometric quantitation of antioxidant capacity through the formation of a phosphomolybdenum complex: specific application to the determination of vitamin E1. Anal. Biochem., 269 (2): 337-341.

Beauchamp C, Fridovich I. 1971. Superoxide dismutase: improved assays and an assay applicable to acrylamide gels. Anal biochem., 44 (1): 276-287.

Braca A, Tommasi N D, Bari L D, Pizza C, Politi M, Morelli I. 2001. Antioxidant principles from Bauhinia terapotensis. J. Nat. Prod., 64: 892-895.

Das S, Dutta M, Chaudhury K, De B. 2016. Metabolomic and chemometric study of Achras sapota L. fruit extracts for identification of metabolites contributing to the inhibition of $\alpha$ amylase and $\alpha$-glucosidase. Eur Food Res Technol., 242 (5): 733 743.

Dasgupta N, De B. 2004. Antioxidant activity of Piper betle L. leaf extract in vitro. Food chem., 88 (2): 219-224.

Feugang J M, Konarski P, Zou D, Stintzing FC, Zou C. 2006. Nutritional and medicinal use of Cactus pear (Opuntia spp.) cladodes and fruits. Front Biosci., 11, 2574-2589.

Gupta M, Sasmal S, Mukherjee A. 2014. Therapeutic effects of acetone extract of Saraca asoca seeds on rats with adjuvantinduced arthritis via attenuating inflammatory responses. ISRN Rheumatol. 1-12.

Hanhineva K, Rogachev I, Kokko H, Mintz-Oron S, Venger I, Karenlampi S, Aharoni A. 2008. Non-targeted analysis of spatial metabolite composition in strawberry ( Fragaria $\mathrm{x}$ ananassa) flowers. Phytochemistry, 69 (13): 2463-2481.

Kelley K M, Behe B K, Biernbaum JA, Poff KL. 2001. Consume preference for edible-flower color, container size, and price. Hort Science., 36: 801-804.

Kind T, Wohlgemuth G, Lee D Y, Lu Y, Palazoglu M, Shahbaz S, Fiehn O. 2009. FiehnLib: mass spectral and retention index libraries for metabolomics based on quadrupole and time-of-flight gas chromatography/mass spectrometry. Anal. Chem., 81: 1003810048 .

Lefer D J, Granger DN. 2000. Oxidative stress and cardiac disease. Am J Med., 109 (4): 315-323.

Marrelli M, Menichini F, Statti GA, Bonesi M, Duez P, Menichini F, Conforti F. 2012.Changes in the phenolic and lipophilic composition, in the enzyme inhibition and antiproliferative activity of Ficus carica L. cultivar Dottato fruits during maturation. Food Chem Toxicol., 50 (3-4): 726-733.

Mishra A, Kumar A, Rajbhar N, Kumar A. 2013. Phytochemical and pharmacological importance of Saraca indica. Int. J. Pharm. Chem. Sci., 2: 1009-1013.

Mishra A, Patel M K, Jha B. 2015. Non-targeted metabolomics and scavenging activity of reactive oxygen species reveal the potential of Salicornia brachiata as a functional food. J Funct Foods., 13: 21-31.
Murthy SM, Mamatha B, Shivananda TN. 2008. Saraca asoca an endangered plant. Biomed., 3: 224-228.

Panchawat S, Sisodia SS. 2010. In vitro antioxidant activity of Saraca asoca Roxb. De Wilde stem bark extracts from various extraction processes. Asian J. Pharm. Clin. Res., 3 (3): 231-233.

Pandey S, Patel M K, Mishra A, Jha B. 2015. Physio-biochemical composition and untargeted metabolomics of cumin (Cuminum cyminum L.) make it promising functional food and help in mitigating salinity stress. PLoS One., 10 (12): e0144469.

Patel MK, Mishra A, Jha B. 2016. Non-targeted metabolite profiling and scavenging activity unveil the nutraceutical potential of Psyllium (Plantago ovate Forsk). Front. Plant Sci., 7: 431.

Pires TCSP, Dias MI, Barros L, Barreira JCM, Buelga CS, Ferreira ICFR. 2018. Incorporation of natural colorants obtained from edible flowers in yogurts. $L W T$, 97: 668-675.

Pradhan P, Joseph L, George M, Kaushik N, Chulet R. 2010. Pharmacognostic, phytochemical and quantitative investigation of Saraca asoca leaves. J. Pharm. Res., 3: 776-780

Pradhan P, Joseph L, Gupta V, Chulet R, Arya H, Verma R, Bajpai A. 2009. Saraca asoca (Ashoka): a review. J. Chem. Pharm., 1 (1): 62-71.

Rajkumar V, Guha G, Kumar RA, Mathew L. 2010. Evaluation of Antioxidant Activities of Bergenia ciliate Rhizome. Rec. Nat. Prod., 4 (1): 38-48.

Saha J, Mitra T, Gupta K, Mukherjee S. 2012. Phytoconstituents and HPTLC analysis in Saraca asoca (Roxb) Wilde. Int. J. Pharm. Pharm. Sci., 4 (1): 96-99.

Saha J, Mukherjee S, Gupta K, Gupta B. 2013. High-performance thin-layer chromatographic analysis of antioxidants present in different parts of Saraca asoca (Roxb.) de Wilde. J Pharm Res., 7 (9): 798-803.

Saha S, Islam Z, Islam S, Hassan MF, Hossain MS, Islam SMS. 2018. Determination of antioxidant properties and the bioactive compounds in wheat (Triticum aestivum L.). JJBS, 11(3): 315321.

Shen Q, Zhang B, Xu R, Wang Y, Ding X, Li P. 2010. Antioxidant activity in vitro of the selenium-contained protein from the Se-enriched Bifidobacterium amimalis. Anaerobe, 16 (4): 380-386.

Shukla R, Chakravarty M, Gautam MP. 2008. Indigenous medicine used for treatment of gynecological disorders by tribal of Chhattisgarh, India. J. Med. Plant Res., 2 (12): 356-360.

Tohge T, Fernie AR. 2014. Metabolomics-inspired insight into developmental, environmental and genetic aspects of Tomato fruit chemical compositionand quality. Plant Cell Physiol., 56 (9): 1681-1696.

Wang L, Yen JH, Liang HL, Wu MJ. 2003. Antioxidant effect of methanol extracts from lotus plumule and blossom (Nelumbo nucifera Gertn.). J Food Drug Anal., 11: 60-66.

Zahin M, Aqil F, Ahmad I. 2009. The in vitro antioxidant activity and total phenolic content of four Indian medicinal plants. Int. J. Pharm., 1: 88-95. 